\title{
Trattamenti fogliari con Arthrospira platensis in viticoltura: primi risultati da esperimenti in campo
}

\author{
Linda Salvi ${ }^{*}{ }^{*}$, Eleonora Cataldo $^{1}$, Alberto Niccolai ${ }^{1}$, Liliana Rodolfi ${ }^{1,2}$, Mario R. Tredici ${ }^{1}$, Paolo Storchi ${ }^{3}$, Giovan \\ Battista Mattii ${ }^{1}$ \\ ${ }^{1}$ DAGRI, Università degli Studi di Firenze, Viale delle Idee 30, 50019 Sesto Fiorentino (FI) \\ ${ }^{2}$ Fotosintetica \& Microbiologica S.r.1., Via della Robbia 54, 50132 Firenze \\ ${ }^{3}$ CREA-VE, Viale Santa Margherita 80, 52100 Arezzo
}

\begin{abstract}
Biostimulants are increasingly assuming a key role in viticulture, thanks to the well-known ability to influence the physiological behavior of plants, promoting the quality of the grapes and improving vine response to abiotic stress. Seaweed extracts are among the most used and studied biostimulants, while there are very few cyanobacterial-based biostimulants currently available on the market. This work had the purpose of investigating the effects of an extract of Arthrospira platensis on eco-physiology, water potential, yield and quality of grapes in Vitis vinifera Experiments were conducted in open field (seasons 2017 and 2018) in Tuscany, carrying out foliar treatments with the A. platensis extract 20 days and ten days before the expected harvest. Following the treatments, gas exchanges and water potential were monitored, and berry samples were collected to evaluate the technological and phenolic quality of the grapes. In general, the treatments only marginally influenced gas exchanges and water potential, with diversified results in relation to the seasonal climatic trend (2017 season, hot and drought; 2018 milder season), indicating a more conservative behavior of the treated vines compared to the control vines. In addition, A. platensis always increased the berry weight, maintaining unchanged or improving the technological and phenolic quality of the grapes at harvest compared to the control. Although no univocal response to treatment emerges, the results globally suggest a positive impact of the leaf distribution of A. platensis on the eco-physiology and quanti-qualitative characteristics in $V$. vinifera candidating cyanobacteria for the formulation of new biostimulants.
\end{abstract}

\section{Introduzione}

Una delle sfide moderne della viticoltura è soddisfare le aspettative dei clienti per una fornitura e una qualità del prodotto costanti, nonostante le fluttuazioni stagionali della resa e della composizione dell'uva guidate da condizioni ambientali variabili [1].

Nella vite, la temperatura e la disponibilità di acqua sono fattori determinanti per la resa e gli attributi qualitativi dell'uva di due vendemmie consecutive, poiché la differenziazione floreale e l'inizio-induzione delle infiorescenze dell'annata successiva avvengono nel periodo di germogliamento-allegagione dell'annata corrente [2]. Il calore estremo o le fluttuazioni di calore possono anche portare a un avanzamento fenologico e a un raggiungimento asincrono di maturità tecnologiche $\mathrm{e}$ fenoliche [3]. In questo contesto, i biostimolanti di origine algale possono servire come strumento per potenziare le strategie della vite per contrastare le condizioni di stress abiotico, raggiungendo un equilibrio ottimale tra resa e qualità $[4,5]$. Le microalghe possono essere particolarmente attraenti in quanto possono essere prodotte su terreni marginali e su acqua salata, presentano un'elevata produttività della biomassa, non richiedono erbicidi o pesticidi e possono sintetizzare co-prodotti di alto valore $[6,7]$. In generale, sebbene siano disponibili diverse informazioni sugli effetti dei biostimolanti derivati dalle alghe sullo sviluppo e sulle funzioni di Vitis vinifera ( $V$. vinifera) [8-10], per quanto ne sappiamo, nessuno studio fa riferimento alle conseguenze dell'applicazione di Arthrospira platensis (A. platensis) su $V$. vinifera.

Per questi motivi, questo esperimento pionieristico si è concentrato sugli effetti ad ampio spettro delle applicazioni fogliari di un estratto di A. platensis F\&MC256 sulle risposte fisiologiche di $V$. vinifera, sulla resa e sulla qualità dell'uva di viti coltivate in campo. Al fine di raggiungere questi obiettivi il confronto tra le viti trattate con estratto di $A$. platensis e quelle non trattate è stato messo a punto per due stagioni consecutive, integrando misurazioni fisiologiche e quanti-qualitative della bacca.

\footnotetext{
*Corresponding author: linda.salvi@unifi.it
} 


\section{Materiali e metodi}

La ricerca è stata condotta durante le stagioni vegetative 2017 e 2018 presso l'azienda agricola Villa Montepaldi, che si trova sulle colline di San Casciano Val di Pesa (FI), nella parte settentrionale della nota zona di produzione del Chianti Classico. Il vigneto nel quale è stata effettuata la sperimentazione è stato impiantato nel 1998 con viti di Sangiovese (clone R 24) innestate su 420A, esposto a Sud-Ovest, con sesto d'impianto di 2,8 × 0,9 e forma d'allevamento a controspalliera, potata a cordone speronato. Il suolo è di medio impasto, con tessitura franco-argillosa (argilla 38.8\%; sabbia 23.4\%; limo $37.8 \%$ ), con $\mathrm{pH}$ leggermente alcalino (7.8). Avvalendosi del disegno sperimentale a blocchi randomizzati con 3 ripetizioni per tesi di 3 filari ciascuna, è stato impostato il confronto tra il non trattato (CTRL) e il trattato con un estratto di A. platensis (APE) prodotto da Fotosintetica \& Microbiologica S.r.l. (Italy), distribuito per via fogliare con dose di $3 \mathrm{~g} / \mathrm{L}$ [11]. Il primo trattamento è stato eseguito 20 giorni prima della prevista vendemmia, mentre il secondo è stato effettuato, sulle stesse piante, dieci giorni dopo il primo trattamento. Negli stessi giorni dei trattamenti con $A$. platensis F\&M-C256, le viti non trattate sono state spruzzate con acqua. Dal filare centrale di ogni ripetizione, sono state selezionate e cartellinate 10 viti omogenee/tesi, usate per i rilievi eco-fisiologici, produttivi e per i campionamenti di acini, in tre fasi fenologiche: $t_{0}$ (pre-trattamento), $t_{1}$ (una settimana dopo il primo trattamento) e $t_{2}$ (vendemmia, una settimana dopo il secondo trattamento). Ad ogni rilievo, campionando 15 foglie adulte e sane poste nella fascia mediana della parete dalle piante cartellinate di ogni tesi, sono stati effettuati rilievi di fotosintesi $\left(\mathrm{P}_{\mathrm{n}}, \mu \mathrm{mol} \mathrm{m} \mathrm{m}^{-2} \mathrm{~s}^{-1}\right)$ e conduttanza stomatica $\left(g_{s}, \mathrm{mmol} \mathrm{m} \mathrm{m}^{-2} \mathrm{~s}^{-1}\right)$ con un analizzatore di gas all'infrarosso (Ciras 3, PP Systems, USA), di potenziale idrico di tralcio $\left(\Psi_{\mathrm{m}}, \mathrm{MPa}\right)$ con una camera a pressione (PMS Instrument Co, USA). Inoltre, nelle stesse tre fasi fenologiche, sono stati effettuati campionamenti di 100 acini da ogni ripetizione/tesi per la determinazione degli zuccheri solubili (g/L), dell'acidità titolabile $(\mathrm{g} / \mathrm{L}$ di acido tartarico), del $\mathrm{pH}$ e del peso dell'acino (g).

Alla raccolta, sulle 10 piante cartellinate per ogni ripetizione di ogni tesi sono stati determinati il peso fresco della produzione $(\mathrm{Kg})$, il numero di grappoli/pianta e il peso fresco del grappolo $(\mathrm{g})$.

I dati raccolti sono stati sottoposti all'analisi della varianza a due vie (trattamento e anno) ed ad una via (APE e CTRL) con SPSS Data Editor (IBM, USA). Le differenze statisticamente significative sono state assunte per $\mathrm{P}<0.05$.

\section{Risultati e discussioni}

\subsection{Effetto dell'estratto di $A$. platensis sui parametri eco-fisiologici in Vitis vinifera}

In Tab. 1 sono riassunti i valori medi dei parametri ecofisiologici misurati durante le stagioni 2017 e 2018 . Nel nostro studio, i trattamenti con A. platensis F\&M-C256 hanno influenzato solo marginalmente gli scambi gassosi ed il potenziale idrico. Infatti, differenze significative sono state registrate solo nella conduttanza stomatica nel 2018, con valori minori per APE rispetto a CTRL. Dato che l'acclimatazione alla siccità nella vite è principalmente mediata da cambiamenti nella conduttanza stomatica [11], è probabile che, nelle viti APE, si sia verificata una chiusura stomatica per risparmiare acqua e mantenere il turgore cellulare [11-13]. Gli effetti positivi sulle funzioni fisiologiche delle piante indotte dai trattamenti con A. platensis sono stati precedentemente riportati anche in barbabietola e pomodoro [14, 15].

Tab 1. Valori medi dei parametri eco-fisiologici nelle stagioni 2017 e 2018.

\begin{tabular}{|c|c|c|c|c|}
\hline Parametro & $\begin{array}{l}\text { APE } \\
2017\end{array}$ & $\begin{array}{l}\text { CTRL } \\
2017\end{array}$ & $\begin{array}{l}\text { APE } \\
2018\end{array}$ & $\begin{array}{l}\text { CTRL } \\
2018\end{array}$ \\
\hline 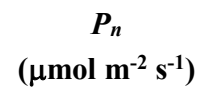 & 5.0 & 5.4 & 5.2 & 5.7 \\
\hline 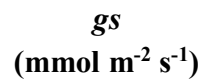 & 76.7 & 82.7 & $151.7 \mathrm{~b}$ & $212.0 \mathrm{a}$ \\
\hline $\begin{array}{c}\text { eWUE } \\
\left(\mu \mathrm{mol} \mathrm{mmol}^{-1}\right)\end{array}$ & 1.8 & 1.9 & 1.6 & 1.4 \\
\hline$\Psi_{m}(\mathbf{M P a})$ & -1.5 & -1.6 & -1.0 & -1.0 \\
\hline
\end{tabular}

Diverse lettere indicano una differenza significativa tra le medie (Anova ad una via, $\mathrm{P}<0.05$ ).

\subsection{Effetto dell'estratto di $A$. platensis sulle caratteristiche qualitative e produttive in Vitis vinifera}

I valori dei parametri tecnologici e fenolici delle uve a vendemmia nelle stagioni 2017 e 2018 sono presentati nella Tab. 2. Non sono state registrate differenze statistiche tra le due tesi nel tenore zuccherino, nel $\mathrm{pH}$ (dati non mostrati) e nel contenuto di antociani totali. Al contrario, differenze significative nell'acidità totale, nel peso di 100 acini e nel contenuto di polifenoli totali sono state osservate a vendemmia tra APE e CTRL. Nel 2018, a piena maturazione, APE presentava acidità maggiore $\mathrm{e}$ contenuto di antociani maggiore rispetto a CTRL. La maggior differenza tra le due tesi in entrambe le annate si è riscontrata nel peso dei 100 acini, sempre considerevolmente maggiore in APE rispetto a CTRL. Questo aumento è stato significativamente più evidente nel 2017 rispetto al $2018(+50 \%$, Tab. 2), quando le elevate temperature e la scarsità d'acqua hanno promosso la disidratazione delle bacche CTRL attraverso la perdita di acqua attraverso il rachide via [16]. Al contrario, in APE gli acini hanno mantenuto un peso più elevato, preservando la loro idratazione probabilmente grazie alla maggiore chiusura stomatica e al controllo del potenziale idrico indotto dall'estratto di A. platensis F\&M-C256.

Per quanto riguarda gli zuccheri nell'acino, nel 2017, APE ha presentato una concentrazione significativamente più alta $(+32 \%)$ rispetto al CTRL al $t_{2}$ (Fig. 1$)$, così come nel 2018 è stato riportato un incremento della concentrazione nelle viti trattate con A. platensis F\&MC256 al t 1 (+ 15\%). Quindi, sia nel 2017 che nel 2018, gli zuccheri ( ${ }^{\circ}$ Brix) sono risultati coerenti tra APE e CTRL, 
mentre, allo stesso tempo, la concentrazione zuccherina (mg per acino) è significativamente superiore in APE. L'ipotesi è che l'estratto di $A$. platensis F\&M-C256 possa aver influenzato il metabolismo degli zuccheri e che il maggiore accumulo di zuccheri nelle bacche APE (ovvero, la maggiore concentrazione zuccherina per acino) possa essere legato all'attitudine delle piante trattate a conservare maggiormente le risorse idriche.

Tab 2. Valori dei parametri tecnologici e fenolici delle uve a vendemmia nelle stagioni 2017 e 2018.

\begin{tabular}{|c|c|c|c|c|}
\hline Parametro & APE 2017 & CTRL 2017 & APE 2018 & CTRL 2018 \\
\hline Zuccheri (g/L) & $249 \pm 7$ & $253 \pm 7$ & $241 \pm 3$ & $236 \pm 2$ \\
\hline Acidità totale (g/L ac. tartarico) & $5.0 \pm 0.1$ & $5.1 \pm 0.1$ & $8.1 \pm 0.4 \mathrm{a}$ & $7.4 \pm 0.1 \mathrm{~b}$ \\
\hline Peso 100 acini (g) & $150 \pm 30 \mathrm{a}$ & $100 \pm 10 \mathrm{~b}$ & $240 \pm 10 \mathrm{a}$ & $210 \pm 10 \mathrm{~b}$ \\
\hline Antociani totali (mg/L) & $1583 \pm 19$ & $1566 \pm 20$ & $1333 \pm 81$ & $1314 \pm 80$ \\
\hline Polifenoli totali (mg/L) & $2333 \pm 62$ & $2266 \pm 90$ & $2437 \pm 26 \mathrm{a}$ & $2292 \pm 42 \mathrm{~b}$ \\
\hline
\end{tabular}

Diverse lettere indicano una differenza significativa tra le medie (ANOVA ad una via; $\mathrm{P}<0.05$ ).

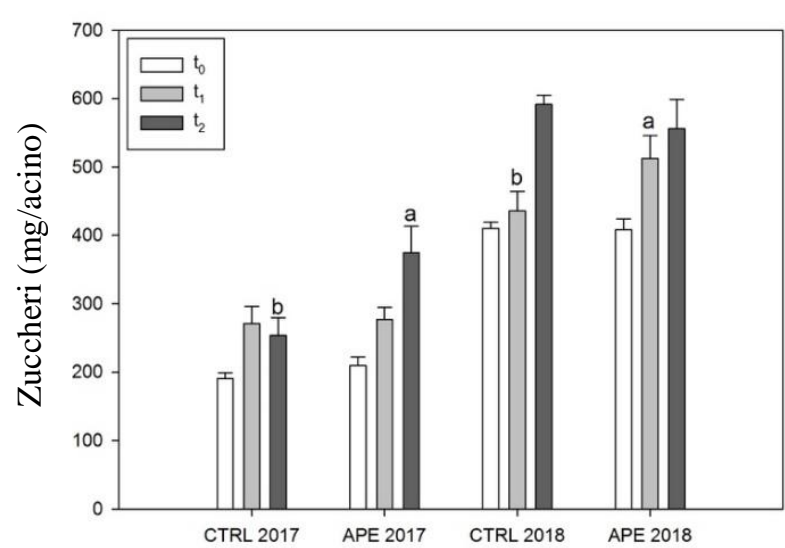

Fig 1. Concentrazione degli zuccheri per acino. Diverse lettere $(a, b)$ indicano una differenza significativa (ANOVA ad una via; $\mathrm{P}<0.05)$.

Ipotizziamo che il miglioramento delle prestazioni fisiologiche e qualitative promosse dai trattamenti con $A$. platensis possa essere correlato alla presenza di aminoacidi, proteine funzionali (ficocianina) e composti fenolici all'interno dell'estratto [17].

\section{Conclusioni}

Nell'ottica di ridurre i fenomeni di sfasamento tra maturità tecnologica e fenolica ascrivibili alle interazioni tra i cambiamenti climatici in atto ed alle mutate tecniche di gestione della chioma, la distribuzione fogliare di prodotti biostimolanti può rappresentare una valida pratica agronomica.

I nostri dati indicano che i trattamenti con A. platensis durante le ultime fasi dello sviluppo delle bacche possono potenziare le prestazioni di $V$. vinifera migliorando i parametri eco-fisiologici e influenzando il metabolismo primario e secondario, con conseguente miglioramento della qualità dell'uva.

In dettaglio, i trattamenti con l'estratto di A. platensis hanno solo marginalmente influenzato i parametri ecofisiologici, riducendo la conduttanza stomatica e promosso una maggior conservazione delle risorse idriche. In termini di caratteristiche della bacca, è stata rilevata un'influenza diretta dei trattamenti con $A$. platensis F\&M-C256 sul peso della bacca, sulla concentrazione zuccherina e sulla maturità fenolica in entrambi gli anni. Nel complesso, le bacche APE hanno presentato un peso maggiore, zuccheri per acino e polifenoli totali più elevati rispetto alle bacche CTRL in entrambi gli anni, il che potrebbe essere stato spinto dalle migliori prestazioni fisiologiche delle viti APE. Riassumendo, è possibile affermare che APE ha mostrato una qualità delle uve simile o migliore rispetto al CTRL, unitamente ad un peso dell'acino maggiore, candidando l'estratto di A. platensis F\&M-C256 come strumento pratico in quelle aree danneggiate da stress abiotici, dove il livello quanti-qualitativo desiderato della produzione è difficile da raggiungere.

\section{Bibliografia}

1. V.O. Sadras, M.A. Moran, P.R. Petrie, Oeno One 51(4), (2017) https://doi.org/10.20870/oenoone.2017.51.4.1913

2. M.C. Vasconcelos et al, The flowering process of Vitis vinifera: a review. Am. J. Enol. Vitic. 60(4), 411-434 (2009)

3. Y. Xu, T. Castel, Y. Richard, C. Cuccia, B. Bois. Climate Dynam. 39(7-8):1613-1626 (2012) 
4. A.S. Khan, B. Ahmad, M.J. Jaskani, R. Ahmad, A.U. Malik. Int. J. Agric. Biol. 14, 383-388 (2012).

5. J.S. Craigie. J. Appl. Phyco.1 23, 371-393 (2011)

6. M.R. Tredici, N. Biondi, E. Ponis et al, Advances in microalgal culture for aquaculture feed and other uses. In: New technologies in aquaculture. Woodhead Publishing Limited, pp 610-676 (2009)

7. T.T. Win, G.D. Barone, F. Secundo, P. Fu. Ind Biotechnol 14, 203-211 (2018)

8. T. Frioni, P. Sabbatini, S. Tombesi, J. Norrie, S. Poni, M. Gatti, A. Palliotti. Sci. Hort. 232, 97-106 (2018).

9. L. Salvi, C. Brunetti, E. Cataldo, A. Niccolai, M. Centritto, F. Ferrini, G.B. Mattii. Plant Physiol. Biochem. 139, 21-32 (2019)

10. L. Salvi, C. Brunetti, E. Cataldo, P. Storchi, G.B. Mattii. Appl.Sci.10(13), 4473 (2020)

11. M.M. Chaves, M.M. Oliveira. J. Exp. Bot. 55(407), 2365-84 (2004)

12. A. Skirycz, D. Inzé. Curr. Opin. Biotechnol. 21, 197 203 https://doi.org/10.1016/j.copbio.2010.03.002

(2010)

13. A. Santaniello, A. Scartazza, F. Gresta, E. Loreti, A. Biasone, D. Di Tommaso, A. Piaggesi, P. Perata. Front. Plant Sci. 8, 1362 (2017)

14. Á.F. Mógor, J. de Oliveira Amatussi, G. Mógor, G.B. de Lara. Am. J. Plant Sci. 9(05), 966 (2018) https://doi.org/10.4236/ajps.2018.95074

15. F. Rachidi, R. Benhima, L. Sbabou, H. El Arroussi. Biotechnol Rep 25, e00426 (2020) https://doi.org/10.1016/j.btre.2020.e00426

16. M.A. Matthews, V. Nuzzo. Acta Hortic. 754, 423436 (2007)

17. A. Niccolai, G.C. Zittelli, L. Rodolfi, N. Biondi, M.R. Tredici. Algal Res. 42, $101617 . \quad$ (2019) https://doi.org/10.1016/j.algal.2019.101617 